\title{
Nucleos(t)ide Analogues for Reducing Hepatocellular Carcinoma in Chronic Hepatitis B Patients: A Systematic Review and Meta-Analysis
}

\author{
Xinhui Wang, Xiaoli Liu, Zhibo Dang, Lihua Yu, Yuyong Jiang, Xianbo Wang, and Zhiyun Yang \\ Center of Integrative Medicine, Beijing Ditan Hospital, Capital Medical University, Beijing, China
}

Background/Aims: Studies have shown that nucleos(t)ide analogue (NA) treatment can reduce the risk of hepatocellular carcinoma $(\mathrm{HCC})$ in chronic hepatitis $\mathrm{B}(\mathrm{CHB})$ patients, but it is unclear which NA is most effective. We performed a meta-analysis and systematic review comparing the efficacies of NAs in CHB patients. Methods: We searched literature databases for randomized controlled trials (RCTs) and observational studies that analyzed the hepatic biochemical response, virological response, seroconversion rate, drug resistance rate, and HCC incidence rate in CHB patients treated with NAs. Meta-analyses were performed with RevMan and Stata/SE software. Results: Twelve cohort studies and one RCT were selected, in which entecavir (ETV), lamivudine (LAM), telbivudine (LdT), and/or tenofovir disoproxil fumarate (TDF) were evaluated in CHB patients. The meta-analysis showed that ETV was superior to LAM with regard to the HCC incidence $(p<0.001)$, biochemical response $(p=0.001)$, virological response $(p=0.02)$, and drug resistance $(p<0.001)$, and ETV was superior to LdT with regard to the virological response $(p<0.001)$ and drug resistance $(p<0.001)$. We found no significant difference between ETV and TDF with regard to the HCC incidence $(p=0.08)$, biochemical response $(p=0.39)$, virological response $(p=0.31)$, serological conversion $(p=0.38)$, or drug resistance $(p=0.95)$. NA-treated patients with pre-existing cirrhosis had a 5.49 times greater incidence of HCC than those without cirrhosis $(p<0.001)$. Conclusions: ETV or TDF should be used for long-term first-line monotherapy in CHB patients according to the current guidelines. Standardized protocols are needed for future studies of ETV and TDF to facilitate conclusive comparisons. Patients with cirrhosis are at significantly elevated risk for HCC, despite the benefits of NA treatment. (Gut Liver 2020;14:232247)
Key Words: Hepatitis B, chronic; Carcinoma, hepatocellular; Nucleos(t)ide analogues; Entecavir; Meta-analysis

\section{INTRODUCTION}

Liver cancer is the third leading cause of cancer-related mortality in China, ${ }^{1}$ the world's most populous nation, and the second leading cause of cancer-related mortality worldwide. ${ }^{2}$ Hepatocellular carcinoma (HCC) is the most common primary malignancy of the liver, accounting for $70 \%$ to $90 \%$ of primary liver cancer cases. ${ }^{2,3}$ Hepatitis B virus (HBV) infection is a major risk factor for $\mathrm{HCC}^{4}$ and the incidence of $\mathrm{HCC}$ is highest in areas where HBV infection is endemic., ${ }^{5,6}$ In recent decades, the increased availability of antiviral treatments and HBV vaccines has resulted in reductions in the incidence of $\mathrm{HBV}$ infection in various regions. ${ }^{3,7}$ However, in 2012, approximately 240 million people had chronic hepatitis B (CHB) infection, ${ }^{8}$ and HBV immunization coverage in areas of endemicity has not increased substantially since then, except in Southeast Asia. ${ }^{9}$ Thus, HBVrelated HCC remains a serious threat to public health on a global scale.

Most CHB patients are treated with alpha-interferon and/or a nucleos(t)ide analogue (NA). ${ }^{10}$ Interferon induces the expression of hundreds of genes that enhance the innate immune response against HBV-infected hepatocytes, ${ }^{11}$ whereas currently available NAs act directly to suppress HBV replication by inhibiting viral reverse transcription. ${ }^{12}$ The use of interferon in clinical practice is, however, often limited because it has severe side effects, which include thrombocytopenia, neutropenia, lymphocytopenia, insomnia, and depression. ${ }^{13,14}$ Side effects of NA treatment are generally mild and infrequent. ${ }^{14}$ At present, NAs approved for treating $\mathrm{CHB}$ worldwide include entecavir (ETV), tenofovir disoproxil fumarate (TDF), tenofovir alafenamide (TAF),

Correspondence to: Zhiyun Yang

Center of Integrative Medicine, Beijing Ditan Hospital, Capital Medical University, No. 8 Jingshun East Street, Chaoyang District, Beijing 100015, China

Tel: +86-10-84322148, Fax: +86-10-84322148, E-mail: Yangzhiyun020116@163.com

Received on December 10, 2018. Revised on March 12, 2019. Accepted on April 5, 2019. Published online October $18,2019$.

pISSN 1976-2283 eISSN 2005-1212 https://doi.org/10.5009/gnl18546

Xinhui Wang and Xiaoli Liu contributed equally to this work as first authors.

(c) This is an Open Access article distributed under the terms of the Creative Commons Attribution Non-Commercial License (http://creativecommons.org/licenses/by-nc/4.0) which permits unrestricted non-commercial use, distribution, and reproduction in any medium, provided the original work is properly cited. 
lamivudine (LAM), adefovir dipivoxil (ADV), and telbivudine (LdT), among which ETV and TDF are recommended for firstline treatment because of the lower incidences of resistance observed with ETV and TDF, compared to other NAs. ${ }^{14,15}$ Continuous treatment with NAs can delay clinical progression of $\mathrm{CHB}$ in patients with and without cirrhosis. ${ }^{16-18}$ However, while NAs suppress viral replication, they do not completely eliminate HBV in many patients. ${ }^{19,20}$ Therefore, many CHB patients need longterm antiviral treatment. ${ }^{14,15,21}$

Clinical studies have shown that NA treatment also reduces the risk of HCC in CHB patients to varying degrees. ${ }^{16,22-26}$ However, many studies have not found significant differences between different NAs with regard to reductions in HCC incidence, ${ }^{27-32}$ except in patients with pre-existing cirrhosis. ${ }^{24,33}$ Direct comparisons between studies of different NAs are confounded by differences in study design, selection methods, treatment regimens, and follow-up durations. ${ }^{34}$ Relatively few large-scale studies have been performed that evaluated the efficacies of different NAs for reducing HCC risk, most of which have consisted of retrospective analyses, ${ }^{26,27}$ which have an inherently higher risk of selection bias than a prospective study or randomized control trial (RCT). Furthermore, a number of studies comparing the efficacy of different NAs for reducing HCC incidence, including one RCT, ${ }^{28}$ have analyzed data from relatively small samples, ${ }^{32,35-37}$ which might call into question the statistical power of the findings from each.

Given that long-term treatment is suggested for many CHB patients, the choice of which NA to use in such circumstances should, of course, consider the patient's biochemical response to antiviral treatment, virological response, seroconversion, nephrotoxicity, and NA resistance, properties which have been widely studied. However, the relative efficacies of NAs for longterm reduction of HCC risk and the effects of cirrhosis on HCC outcomes in NA-treated CHB patients are topics that have not been evaluated thoroughly. To facilitate evidence-based selection of NAs for long-term antiviral treatment in CHB patients, we performed a systematic review and meta-analysis of investigations of NA efficacy that examined HCC incidence as a primary outcome in patients with or without pre-existing cirrhosis. Given that ETV is likely the most widely used first-line antiviral treatment for CHB currently, we performed our analysis using ETV as the reference against which other NAs were compared.

\section{MATERIALS AND METHODS}

\section{Selection criteria}

We included RCTs and observational studies that met the following inclusion criteria: (1) Language: published in English. (2) Patients: adults who had received a diagnosis of CHB based on HBsAg-positive lab results or a documented history of HBV infection for $\geq 6$ months, with or without cirrhosis; were either treatment naive or had previously received NAs; and if previ- ously treated with an NA, had no drug resistance at enrolment. (3) Intervention: one treatment group receiving ETV and at least one other group receiving TDF, TAF, LAM, ADV, or LdT; and a minimum treatment duration of 3 months for all groups. (4) Comparator: ETV treatment group. (5) Outcomes: the primary outcome examined was HCC incidence at $\geq 1$ year following initiation of NA treatment. We excluded studies that included patients who developed HCC or died within the first 6 months of treatment and those that included patients who received medications that might affect HCC risk, such as interferon, metformin, statins, or antiplatelet agents.

Secondary outcomes included hepatic biochemical response, virological response, seroconversion, antiviral drug resistance, and development of cirrhosis. Favorable biochemical response was defined as the normalization of the level of alanine aminotransferase as assessed by routine hepatic panel. We defined early cirrhosis as histological findings (Ishak score 5-6) or radiological findings in the absence of decompensated hepatic function or portal hypertension. Favorable virological response was defined as the loss of hepatitis B e antigen ( $\mathrm{HBeAg}$ ) with or without anti-HBe antibodies and undetectable HBV DNA, or undetectable HBV DNA in an HBeAg-negative patient. Antiviral drug resistance was defined as the reappearance of HBV DNA after a period of non-detectable HBV DNA. We excluded articles with previously published data, meta-analyses, review articles, editorials and other types of commentary articles, conference abstracts and presentations, and any publication from which data regarding HCC incidence could not be extracted. Studies that lacked clear definitions of biochemical response, virological response, drug resistance, and cirrhosis were excluded from the respective subgroup analysis.

\section{Data sources and searches}

On December 31, 2018, we performed separate searches of MEDLINE, Embase, and Cochrane Library using the following terms: (1) "[hepatocellular carcinoma OR liver cell carcinoma OR liver cancer OR hepatoma] AND [antiviral OR antiviral drug OR nucleoside analogue $\mathrm{OR}$ nucleotide analogue OR nucleos(t)ide analogue]"; (2) "[entecavir OR Baraclude] AND [tenofovir disoproxil fumarate OR tenofovir disoproxil OR tenofovir OR Viread OR tenofovir alafenamide OR Vemlidy] AND [hepatocellular carcinoma OR liver cell carcinoma OR liver cancer OR hepatoma]"; (3) "[entecavir OR Baraclude] AND [lamivudine OR Epivir OR 3TC OR Zeffix] AND [hepatocellular carcinoma OR liver cell carcinoma OR liver cancer OR hepatoma]"; (4) "[entecavir OR Baraclude] AND [adefovir OR adefovir dipivoxil OR bis-POM PMEA OR Preveon OR Hepsera] AND [hepatocellular carcinoma OR liver cell carcinoma OR liver cancer OR hepatoma]"; and (5) "[entecavir OR Baraclude] AND [telbivudine OR Sebivo OR Tyzeka] AND [hepatocellular carcinoma OR liver cell carcinoma OR liver cancer OR hepatoma]”. Two researchers (X.W. and Z.D.) independently viewed the titles and abstracts of the articles re- 
trieved, and those that were obviously irrelevant to the selection criteria were discarded.

\section{Study selection and data extraction}

Study selection and data extraction were performed independently by two researchers (X.W. and Z.D.). Titles of the retrieved studies were screen to remove duplicates. Afterward, the abstracts were reviewed to exclude those that did not meet the inclusion criteria. The full-text versions of the remaining articles were then reviewed to determine inclusion/exclusion. For articles in which they were not in agreement, a third researcher (X.L.) was consulted to reach a majority decision. Data extraction was performed using a standardized form, which included the following information: (1) Basic study information included the authors' names, publication date, study location, study dates and duration, sample sizes, and details of the study design, including descriptions of blinding, allocation concealment, and randomization for RCTs. (2) Baseline characteristics of the study subjects included age, sex, ethnicity, serum biochemical data, CHB diagnostic criteria, HBV DNA level, cirrhosis, decompensation of cirrhosis, previous therapy for $\mathrm{CHB}$, and previous NA treatment. (3) Intervention details included the NA drug used, duration of NA treatment, and follow-up period. (4) Outcome measures included the incidence of HCC, development of cirrhosis or decompensation, incidence of NA resistance, serological conversion, and biochemical and virological responses to NA treatment.

\section{Quality assessment}

The methodological quality of the data from selected studies was evaluated based on the risk of various biases involved according to the study design. Methodological quality of data from the cohort studies was assessed using the NewcastleOttawa Scale, by which a total score of 0 to 9 stars was assigned to each study. ${ }^{38}$ A maximum of 4 stars for selection, 2 stars for comparability, and 3 stars for outcome was combined to calculate the total Newcastle-Ottawa Scale score, with the maximum score of 9 stars indicating lowest possible risk of bias. Methodological quality in RCTs was assessed using the Jadad Scale, whereby a maximum score of 5 indicated lowest possible risk of bias. $^{39}$

\section{Statistical analysis}

The Review Manager 5 (RevMan 5) software, version 5.3 (Nordic Cochrane Center, Copenhagen, Denmark) and Stata/SE software, version 15.0 (StataCorp., College Station, TX, USA), were used for the meta-analysis of the selected studies and the presentation of the results. ${ }^{40}$ Comparisons of the outcome incidences are expressed as risk ratio (RR) and 95\% confidence interval (CI), which were calculated using random or fixed effects models, and the results are presented as Forest plots. Heterogeneity was evaluated based on the $\mathrm{I}^{2}$ statistic. A fixed-effects model was used when significant heterogeneity existed among the studies, and a subgroup analysis was conducted to identify factors contributing to heterogeneity. A random-effects model was used when significant heterogeneity was not detected. A

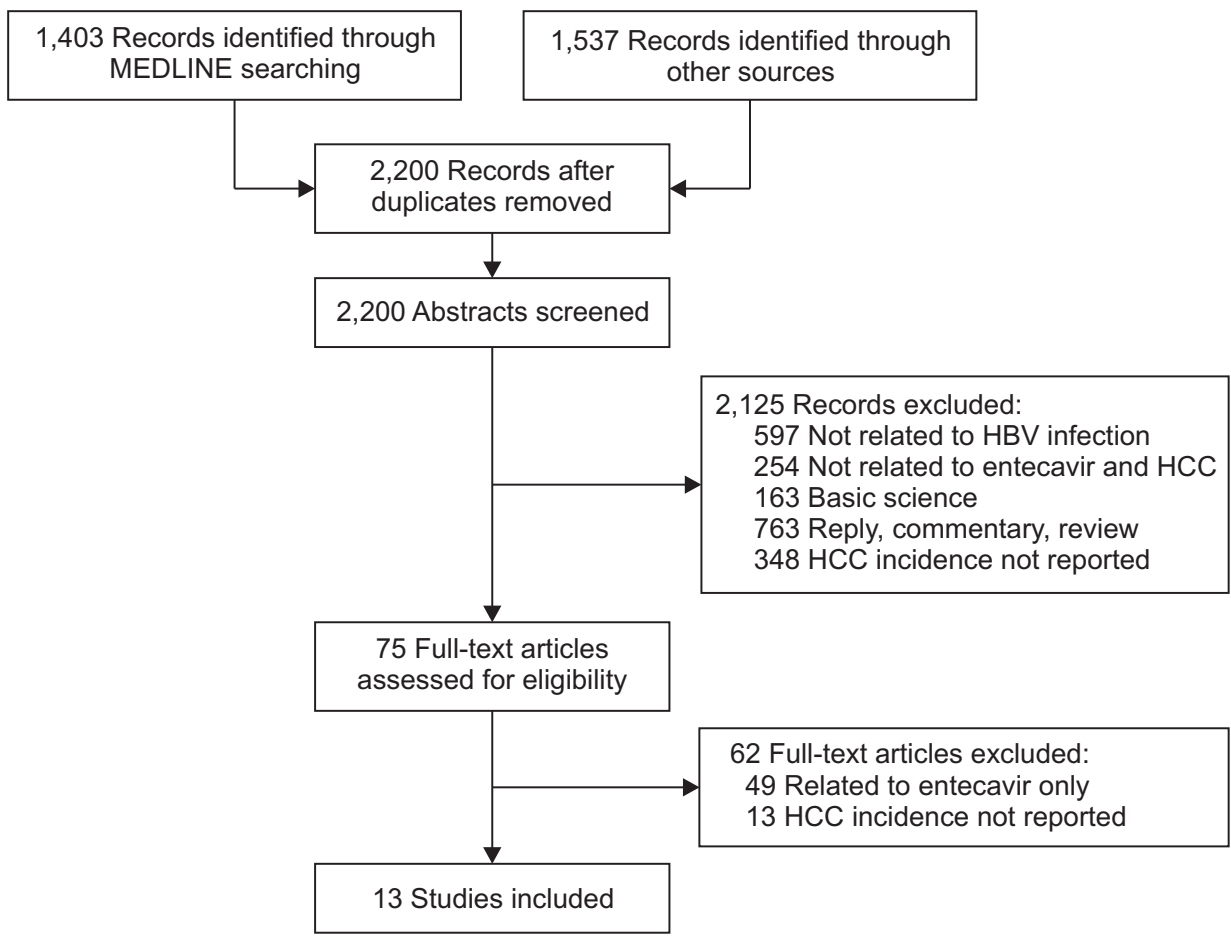

Fig. 1. Flowchart of study selection. HBV, hepatitis B virus; HCC, hepatocellular carcinoma. 
descriptive analysis was used if the source of heterogeneity between two groups could not be eliminated. Heterogeneity between groups was evaluated using a chi-square analysis with $\alpha=0.10$. The effect size was estimated based on the $Z$ statistic, which was evaluated using a chi-square analysis with $\alpha=0.05$. In the case of heterogeneity, a sensitivity analysis was used to apportion the source of heterogeneity by step-wise removal of one of each of the studies in the data set (n-1) over consecutive iterations (n). Funnel plots were used to examine the potential for publication bias, and the Begg's test and Egger's test were performed with a level of significance set at $\mathrm{p}<0.10$.

\section{RESULTS}

\section{Search results and literature screening}

Study selection is depicted in the flow diagram in Fig. 1. A total of 2,940 articles were retrieved in the electronic searches, from which 740 duplicates were removed. In abstract/title screening of the remaining 2,200 articles, we discarded 2,125 articles that did not meet the inclusion criteria. Full-text screen-

Table 1. Basic Characteristics of the Included ETV Studies

\begin{tabular}{|c|c|c|c|c|c|}
\hline Study (year) & Design & Location & Population & Naive & Other NAs \\
\hline Kobashi et al. $(2011)^{30}$ & Cohort & Japan & CHB, LC & Yes & LAM \\
\hline Hosaka et al. $(2013)^{24}$ & Cohort & Japan & CHB, LC & Yes & LAM \\
\hline Köklü et al. (2013) $)^{37}$ & Cohort & Turkey & LC & Partly & LAM, TDF \\
\hline Tsai et al. $(2014)^{32}$ & Cohort & Taiwan & CHB, LC & Yes & LdT \\
\hline Lim et al. $(2014)^{27}$ & Cohort & Korea & CHB, LC & Unknown & LAM \\
\hline Idilman et al. $(2015)^{29}$ & Cohort & Turkey & $\mathrm{CHB}$ & Yes & TDF \\
\hline Hsu et al. $(2014)^{36}$ & Cohort & Taiwan & CHB, LC & Unknown & LAM, LdT, TDF \\
\hline Coffin et al. (2014) & Cohort & Canada & CHB, LC & Unknown & LAM, LdT, ADV, TDF \\
\hline Papatheodoridis et al. (2015) ${ }^{33}$ & Cohort & Greece & $\mathrm{CHB}, \mathrm{LC}$ & Yes & LAM \\
\hline Riveiro-Barciela et al. (2017) ${ }^{31}$ & Cohort & Spain & CHB, LC & Yes & TDF \\
\hline An et al. $(2017)^{28}$ & $\mathrm{RCT}$ & Korea & CHB, LC & Yes & LdT \\
\hline Choi et al. (2018) ${ }^{41}$ & Cohort & Korea & CHB, LC & Yes & TDF \\
\hline Kim et al. (2018) ${ }^{42}$ & Cohort & Korea & CHB, LC & Yes & TDF \\
\hline
\end{tabular}

ETV, entecavir; NA, nucleos(t)ide analogue; CHB, chronic hepatitis B; LC, liver cirrhosis; LAM, lamivudine; TDF, tenofovir disoproxil fumarate; $\mathrm{LdT}$, telbivudine; ADV, adefovir dipivoxil; RCT, randomized control trial.

Table 2. Risk of Bias Analysis

\begin{tabular}{|c|c|c|c|}
\hline \multirow{2}{*}{ Study (year) } & \multicolumn{3}{|c|}{ Study quality } \\
\hline & Selection & Comparability & Outcome/exposure \\
\hline \multicolumn{4}{|l|}{ Cohort study } \\
\hline Coffin et al. (2014) $)^{35}$ & $* * *$ & - & $* *$ \\
\hline Hosaka et al. $(2013)^{24}$ & $* * * *$ & $* *$ & $* * *$ \\
\hline Hsu et al. (2014) & $* * *$ & - & $* *$ \\
\hline Idilman et al. $(2015)^{29}$ & $* * * *$ & - & * \\
\hline Kobashi et al. $(2011)^{30}$ & $* * * *$ & $*$ & $*$ \\
\hline Köklü et al. (2013) & $* * *$ & - & $*$ \\
\hline Lim et al. $(2014)^{27}$ & $* * *$ & $* *$ & $* *$ \\
\hline Papatheodoridis et al. (2015) & $* * *$ & $*$ & $* *$ \\
\hline Riveiro-Barciela et al. $(2017)^{31}$ & $* * *$ & $*$ & $* *$ \\
\hline Tsai et al. $(2014)^{32}$ & $* * * *$ & $* *$ & $* *$ \\
\hline Choi et al. (2018) ${ }^{41}$ & $* * *$ & $* *$ & $* * *$ \\
\hline Kim et al. (2018) ${ }^{42}$ & $* * *$ & $* *$ & $* * *$ \\
\hline RCT & Randomized & Double-blind & Withdrawals/dropouts \\
\hline An et al. $(2017)^{28}$ & 1 & 1 & - \\
\hline
\end{tabular}

$\mathrm{RCT}$, randomized control trial. 
ing of the remaining 75 articles resulted in the exclusion of 62 articles, leaving a total of 13 articles that were selected for meta-analysis. No studies of TAF met the inclusion criteria.

\section{Study characteristics and quality}

The basic characteristics of the selected studies are shown in Table 1. A total of 13 studies were selected for meta-analysis, which included 12 cohort studies ${ }^{24,27,29-33,35-37,41,42}$ and 1 RCT. $^{28}$ Only one study ${ }^{35}$ examined the effects of ADV treatment on HCC incidence. The study locations included Korea $(n=4)$, Japan $(\mathrm{n}=2)$, Taiwan $(\mathrm{n}=2)$, Turkey $(\mathrm{n}=2)$, Canada $(\mathrm{n}=1)$, Spain $(\mathrm{n}=1)$, and Greece $(n=1)$. Most of study participants were of Asian or Caucasian ethnicity. The results of bias risk assessment are also shown in Table 2. Only one cohort study ${ }^{24}$ scored 9 stars on the Newcastle-Ottawa Scale, whereas four of the cohort studies ${ }^{29,35-37}$ had a total score of 5 stars or less. The single RCT selected for our meta-analysis scored 2 on the Jadad Scale. Data regarding ADV-treated patients ${ }^{35}$ was deemed insufficient for metaanalysis due to small sample size $(n=11)$.

\section{HCC incidence in NA-treated CHB patients}

In our initial meta-analysis, we compared the incidence of HCC in CHB patients treated with ETV with that of patients treated with other NAs. Treatment with ETV reduced the incidence of HCC by $21 \%$ (RR, 0.79; 95\% CI, 0.49 to 1.27 ; $=0.34$ ), compared with that of other NAs, but the difference was not

A

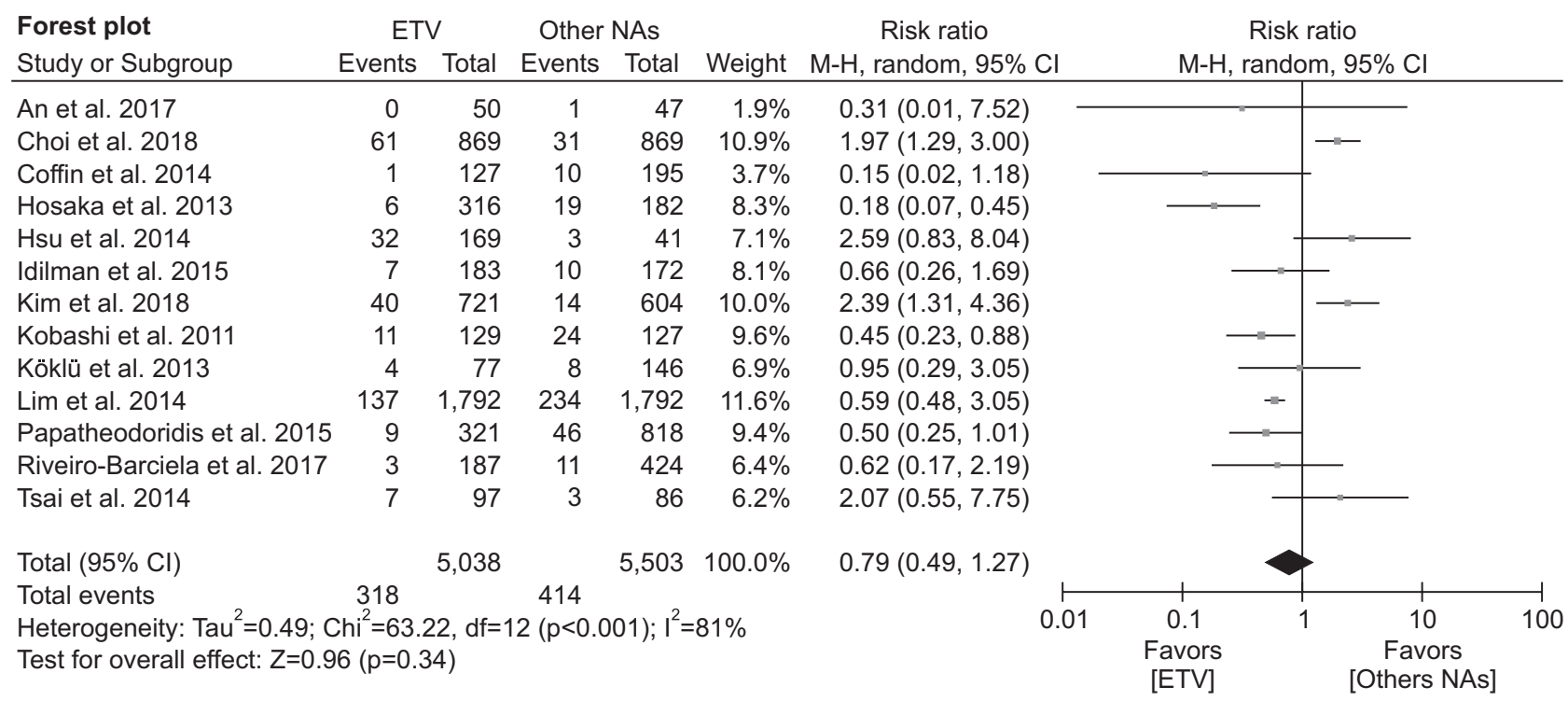

\section{Funnel plot}

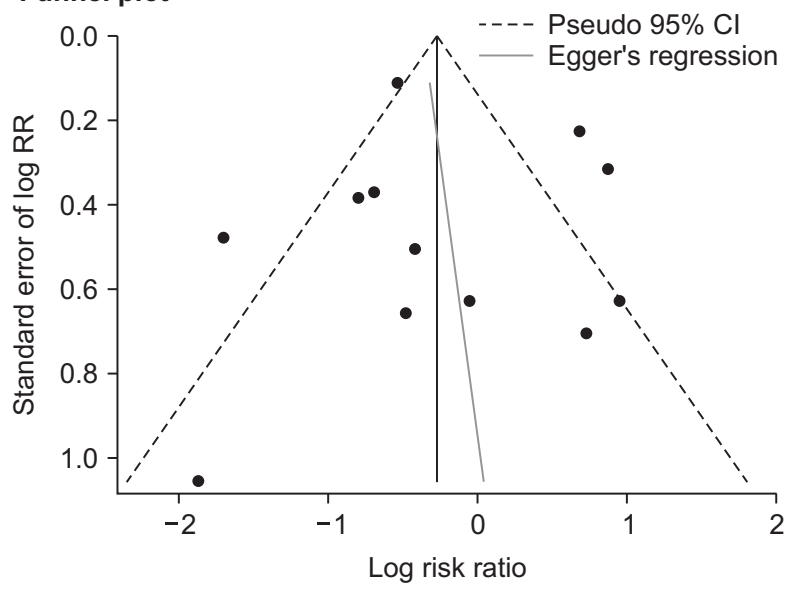

Fig. 2. (A) Forest and funnel plots for the comparison of hepatocellular carcinoma (HCC) incidence between entecavir (ETV)-treated chronic hepatitis $B(C H B)$ patients and those treated with other NA antiviral drugs (Begg's test: $z=-0.07, p=1.000$; Egger's test: $t=0.34$, $p=0.743$ ). (B) Forest and funnel plots for the comparison of HCC incidence between ETV and LAM (Begg's test: $z=-0.30, p=0.764$; Egger's test: $t=-1.51, p=0.191$ ). (C) Forest plot for the comparison of HCC incidence between ETV and LdT. (D) Forest and funnel plots for the comparison of HCC incidence between ETV and TDF (Begg's test: $\mathrm{z}=-0.60, \mathrm{p}=0.540$; Egger's test: $\mathrm{t}=-1.78, \mathrm{p}=0.135$ ).

NA, nucleos(t)ide analogue; LAM, lamivudine; LdT, telbivudine; TDF, tenofovir disoproxil fumarate; M-H, Mantel-Haenszel; CI, confidence interval; RR, risk ratio. 
B

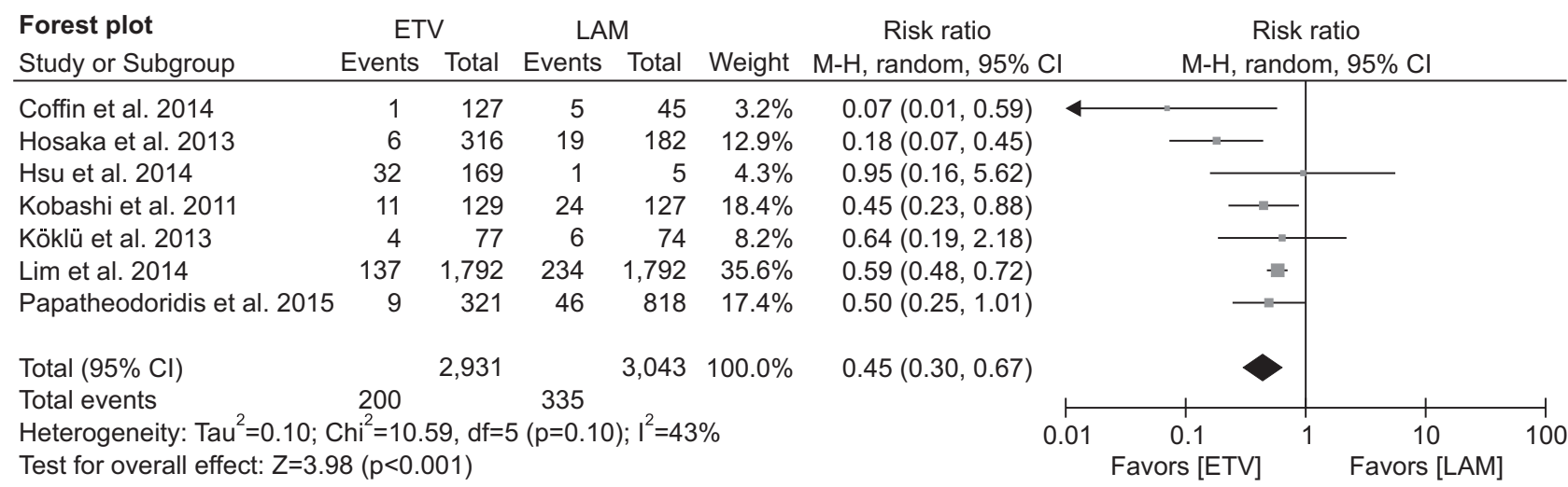

Funnel plot

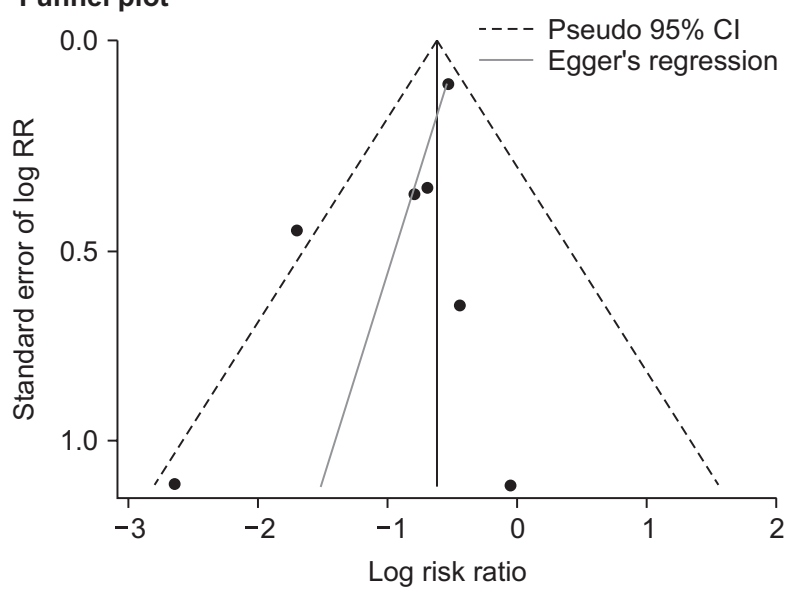

C

\begin{tabular}{|c|c|c|c|c|c|c|c|c|c|}
\hline Forest plot & ET & & 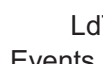 & & & Risk ratio & Ris & k ratio & \\
\hline Study or Subgroup & Events & Total & Events & Total & Weight & $\mathrm{M}-\mathrm{H}$, random, 95\% Cl & & & \\
\hline An et al. 2017 & 0 & 50 & 1 & 47 & $24.7 \%$ & $0.31(0.01,7.52)$ & & & \\
\hline Coffin et al. 2014 & 1 & 127 & 0 & 5 & $15.3 \%$ & $0.14(0.01,3.10)$ & & & \\
\hline Hsu et al. 2014 & 32 & 169 & 2 & 11 & $60.0 \%$ & $1.04(0.29,3.79)$ & & & \\
\hline Total $(95 \% \mathrm{Cl})$ & & 346 & & 63 & $100.0 \%$ & $0.72(0.24,2.14)$ & & & \\
\hline Total events & 33 & & 3 & & & & & & \\
\hline $\begin{array}{l}\text { Heterogeneity: } \mathrm{Chi}^{2} \\
\text { Test for overall effec }\end{array}$ & $\begin{array}{l}\mathrm{ff}=2(p=0 \\
58(p=0.5\end{array}$ & $\begin{array}{l}\text {.44); } 1^{2} \\
6)\end{array}$ & $=0 \%$ & & & 0.01 & $\begin{array}{cc}1 & 0.1 \\
\text { Favors [ETV] }\end{array}$ & $\begin{array}{r}1 \\
\text { Favors }\end{array}$ & $\begin{array}{l}10 \\
\mathrm{rs}[\mathrm{LdT}]\end{array}$ \\
\hline
\end{tabular}

Fig. 2. Continued 1.

statistically significant (Fig. 2A). However, significant heterogeneity in HCC incidence data was detected among the 13 studies $\left(\mathrm{I}^{2}=81 \%, \mathrm{p}<0.001\right)$, so subgroup analyses of the different NAs were performed. In the seven studies in which LAM was used, ${ }^{24,27,30,33,35-37}$ while heterogeneity persisted $\left(I^{2}=43 \%, p=0.10\right)$, the HCC incidence in CHB patients treated with ETV was 55\% lower than that of those who were treated with LAM (RR, 0.45; $95 \%$ CI, 0.30 to $0.67 ; \mathrm{p}<0.001)$. A sensitivity analysis was performed to identify the source of heterogeneity, but did not provide a statistically significant result (data not shown). The incidence of HCC in ETV-treated CHB patients was lower than that in LdT-treated patients ${ }^{28,35,36}$ (RR, 0.72; 95\% CI, 0.24 to 2.14) (Fig.
2C), but was higher than that in TDF-treated CHB patients ${ }^{35,36}$ (RR, 1.52; 95\% CI, 0.94 to 2.44) (Fig. 2D). However, these differences in HCC incidence were not statistically significant ( $>0.05$ for both). These results were undoubtedly influenced by heterogeneity in the two data sets. While heterogeneity in the ETV versus LdT and ETV versus TDF subgroup analyses was less than that in the overall analysis, the results were not statistically significant ( $p>0.05$ for both). A subgroup analysis of ETV versus $A D V$ was not performed due to the small sample of ADVtreated patients $(n=11)$ in the Coffin et al. ${ }^{35}$ study, which was the only study that used ADV among the 13 studies included in our meta-analysis. Funnel plots for the overall (Fig. 2A), ETV versus 
D

\begin{tabular}{|c|c|c|c|c|c|c|c|c|}
\hline $\begin{array}{l}\text { Forest plot } \\
\text { Study or Subgroup }\end{array}$ & \multicolumn{2}{|c|}{ ETV } & \multicolumn{2}{|c|}{ TDF } & \multicolumn{2}{|r|}{ Risk ratio } & \multicolumn{2}{|c|}{$\begin{array}{c}\text { Risk ratio } \\
\mathrm{M}-\mathrm{H} \text {, random, } 95 \% \mathrm{Cl}\end{array}$} \\
\hline Choi et al. 2018 & 61 & 869 & 31 & 869 & $28.7 \%$ & $1.97(1.29,3.00)$ & & $-\square$ \\
\hline Coffin et al. 2014 & 1 & 127 & 3 & 132 & $4.0 \%$ & $0.35(0.04,3.29)$ & & \\
\hline Hsu et al. 2014 & 32 & 169 & 0 & 25 & $2.7 \%$ & $9.94(0.63,157.44)$ & & \\
\hline Idilman et al. 2015 & 7 & 183 & 10 & 172 & $15.0 \%$ & $0.66(0.26,1.69)$ & & - \\
\hline Kim et al. 2018 & 40 & 721 & 14 & 604 & $23.3 \%$ & $2.39(1.31,4.36)$ & & 一- \\
\hline Köklü et al. 2013 & 4 & 77 & 2 & 72 & $6.6 \%$ & $1.87(0.35,9.90)$ & & \\
\hline Riveiro-Barciela et al. 2017 & 3 & 187 & 11 & 424 & $10.2 \%$ & $0.62(0.17,2.19)$ & & - \\
\hline Tsai et al. 2014 & 7 & 97 & 3 & 86 & $9.5 \%$ & $2.07(0.55,7.75)$ & & \\
\hline Total $(95 \% \mathrm{Cl})$ & & 2,430 & & 2,384 & $100.0 \%$ & $1.52(0.95,2.44)$ & & \\
\hline Total events & 155 & & 74 & & & & & \\
\hline $\begin{array}{l}\text { Heterogeneity: } \mathrm{Tau}^{2}=0.16 ; \\
\text { Test for overall effect: } Z=1.7\end{array}$ & $\begin{array}{l}C h i^{2}=11 . \\
73(p=0 . C\end{array}$ & $\begin{array}{l}\text { 69, } d f=7 \\
08)\end{array}$ & $p=0.1$ & ${ }^{2}=$ & & 0.01 & $\begin{array}{c}0.1 \\
\text { Favors [ETV] }\end{array}$ & $\begin{array}{r}1 \\
\text { Favors }\end{array}$ \\
\hline
\end{tabular}

\section{Funnel plot}

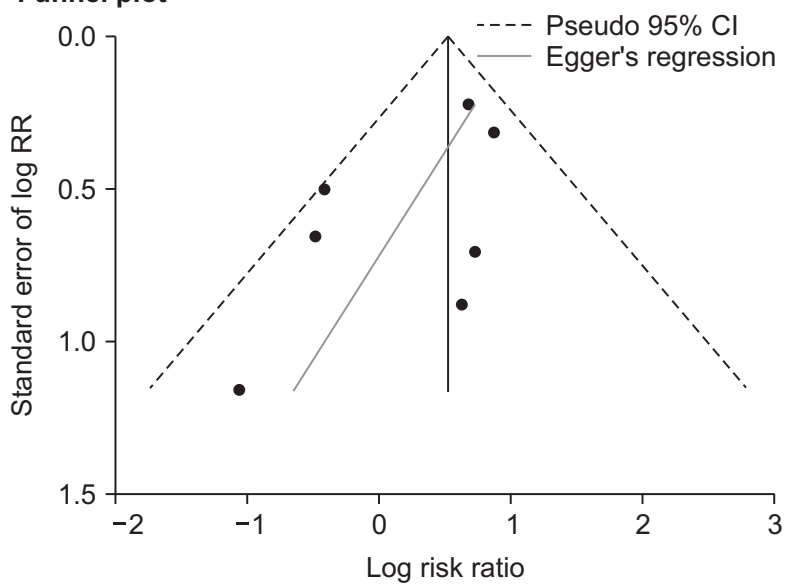

Fig. 2. Continued 2.

LAM (Fig. 2B), and ETV versus TDF (Fig. 2D) subgroup analyses did not indicate publication bias (Begg's and Egger's tests: $p>0.1$ for all). No funnel plot was constructed for ETV versus LdT because only three studies were analyzed.

\section{Biochemical response in NA-treated CHB patients}

In the three studies ${ }^{31,32,37}$ that stringently examined biochemical response to NA treatment, treatment with ETV increased the incidence of favorable biochemical response in CHB patients by 12\% (RR, 1.12; 95\% CI, 1.01 to $1.24 ; \mathrm{p}=0.04$ ), compared with that observed in patients treated with LAM, TDF, or LdT (Fig. 3A). However, significant heterogeneity was detected among the three studies $\left(I^{2}=53 \%, p=0.10\right)$. In the subgroup analyses (Fig. 3A), ETV treatment significantly increased the incidence of favorable biochemical response in CHB patients (RR, 1.32; 95\% CI, 1.11 to $1.56 ; p=0.001$ ), compared to LAM treatment, ${ }^{37}$ whereas no significant difference was observed in the incidence of favorable biochemical response between ETV-treated CHB patients and those treated with $\mathrm{TDF}^{31,37}$ (RR, 1.06; 95\% CI, 0.93 to $1.20 ; \mathrm{p}=0.39$ ) or those treated with $\mathrm{LdT}^{32}$ (RR, 1.09; 95\% CI, 0.96 to $1.23 ; \mathrm{p}=0.17$ ). Although the Begg's test of the funnel plot data indicated potential publication bias $(\mathrm{p}=0.089)$ (Fig,
3A), it was likely confounded by the small number of studies in the analysis of biochemical response $(n=3)$, whereas the Egger's test did not indicate publication bias $(\mathrm{p}=0.119)$ (Fig. 3A). Funnel plots were not constructed for the subgroup analyses due to the small number of studies in each.

\section{Virological response in NA-treated CHB patients}

Six of the selected studies ${ }^{24,28,31,32,37,41}$ examined virological response in CHB patients treated with NAs. Among these studies, treatment with ETV increased the incidence of favorable virological response by $13 \%$ (RR, 1.13 ; 95\% CI, 0.99 to 1.30 ; $\mathrm{p}=0.08$ ), compared with that in patients treated with LAM, LdT, or TDF (Fig. 3B), but the difference was not statistically significant. Significant heterogeneity was detected among these studies $\left(I^{2}=91 \%, p<0.001\right)$. Subgroup analyses showed that rate of favorable virological response for ETV was 15\% greater than that for LAM (RR, 1.15; 95\% CI, 1.03 to 1.29 ; $\mathrm{p}<0.02$ ), and $37 \%$ greater than that for LdT (RR, 1.37; 95\% CI, 1.16 to 1.62 ; $\mathrm{p}<0.001)$. The rate of favorable virological response in ETVtreated patients versus that in TDF-treated patients was statistically similar (RR, 0.95; 95\% CI, 0.86 to $1.05 ; \mathrm{p}=0.31$ ). However, both the Begg's and Egger's tests of the funnel plot data indi- 
A

\begin{tabular}{|c|c|c|c|c|c|c|c|c|}
\hline $\begin{array}{l}\text { Forest plot } \\
\text { Study or Subgroup }\end{array}$ & \multicolumn{2}{|c|}{ ETV } & \multicolumn{2}{|c|}{ Control } & Weight & Risk ratio & \multicolumn{2}{|c|}{$\begin{array}{c}\text { Risk ratio } \\
\mathrm{M}-\mathrm{H} \text {, random, } 95 \% \mathrm{Cl}\end{array}$} \\
\hline \multicolumn{9}{|l|}{ LAM } \\
\hline Köklü et al. 2013 & 70 & 77 & 51 & 74 & $21.4 \%$ & $1.32(1.11,1.56)$ & & - \\
\hline Subtotal $(95 \% \mathrm{Cl})$ & & 77 & & 74 & $21.4 \%$ & $1.32(1.11,1.56)$ & & 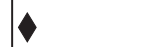 \\
\hline Total events & 70 & & 51 & & & & & \\
\hline \multicolumn{9}{|c|}{ Heterogeneity: Not applicable } \\
\hline \multicolumn{9}{|c|}{ Test for overall effect: $Z=3.22(p=0.001)$} \\
\hline \multicolumn{9}{|l|}{ LdT } \\
\hline Tsai et al. 2014 & 98 & 115 & 90 & 115 & $28.9 \%$ & $1.09(0.96,1.23)$ & & 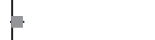 \\
\hline Subtotal $(95 \% \mathrm{Cl})$ & & 115 & & 115 & $28.9 \%$ & $1.09(0.96,1.23)$ & & $b$ \\
\hline Total events & 98 & & 90 & & & & & \\
\hline \multicolumn{9}{|c|}{ Heterogeneity: Not applicable } \\
\hline \multicolumn{9}{|c|}{ Test for overall effect: $Z=1.36(p=0.17)$} \\
\hline \multicolumn{9}{|l|}{ TDF } \\
\hline Köklü et al. 2013 & 70 & 77 & 59 & 72 & $27.7 \%$ & $1.11(0.97,1.26)$ & & $=$ \\
\hline Riveiro-Barciela et al. 2017 & 97 & 187 & 222 & 424 & $21.9 \%$ & $0.99(0.84,1.17)$ & & 1 \\
\hline Subtotal $(95 \% \mathrm{Cl})$ & & 264 & & 496 & $49.6 \%$ & $1.06(0.93,1.20)$ & & $\gamma$ \\
\hline Total events & 167 & & 281 & & & & & \\
\hline \multicolumn{9}{|c|}{ Heterogeneity: $\operatorname{Tau}^{2}=0.00 ; \mathrm{Chi}^{2}=1.54, \mathrm{df}=1(p=0.22) ; I^{2}=35 \%$} \\
\hline \multicolumn{9}{|c|}{ Test for overall effect: $Z=0.86(p=0.39)$} \\
\hline Total $(95 \% \mathrm{Cl})$ & & 456 & & 685 & $100.0 \%$ & $1.12(1.01,1.24)$ & & b \\
\hline Total events & 335 & & 422 & & & & 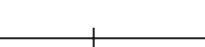 & \\
\hline \multirow{2}{*}{\multicolumn{8}{|c|}{ Heterogeneity: $\operatorname{Tau}^{2}=0.01 ; \mathrm{Chi}^{2}=6.32, \mathrm{df}=3(\mathrm{p}=0.10) ; I^{2}=53 \%$}} & 1 \\
\hline & & & & & & & & Favors [c \\
\hline
\end{tabular}

Test for subgroup differences: $\mathrm{Chi}^{2}=4.58, \mathrm{df}=2(\mathrm{p}=0.10) ; \mathrm{I}^{2}=56.3 \%$

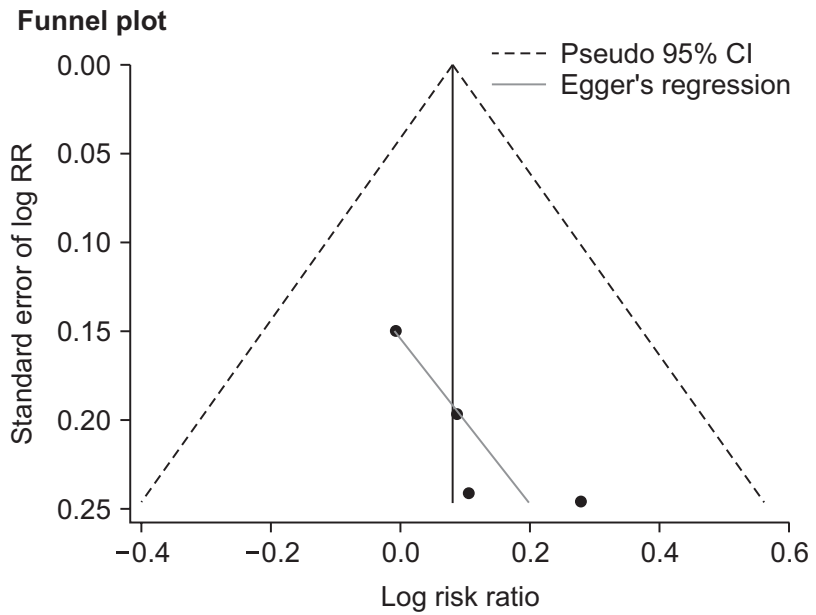

Fig. 3. (A) Forest and funnel plots for the comparison of the biochemical response in entecavir (ETV)-treated chronic hepatitis B (CHB) patients and CHB patients treated with lamivudine (LAM), telbivudine (LdT), or tenofovir disoproxil fumarate (TDF) as controls (Begg's test: $\mathrm{z}=1.70$, $\mathrm{p}=0.089$; Egger's test: $t=-2,64, \mathrm{p}=0.119$ ). (B) Forest and funnel plots for the comparison of the virological response in CHB patients treated with ETV and those treated with LAM, LdT, or TDF as controls (Begg's test: $\mathrm{z}=1.00, \mathrm{p}=0.072$; Egger's test: $t=4.02, \mathrm{p}=0.010$ ). (C) Forest and funnel plots for the comparison of the serological conversion in CHB patients treated with ETV and those treated with LAM, LdT, or TDF as controls (Begg's test: $\mathrm{z}=0.00$; $\mathrm{p}=1.000$; Egger's test: $\mathrm{t}=-1.23, \mathrm{p}=0.436$ ). (D) Incidence of drug resistance in CHB patients treated with ETV compared with that in those treated with LAM, LdT, or TDF as controls (Begg's test: $z=0.75$, $p=0.452$; Egger's test: $t=0.99, p=0.380$ ).

$\mathrm{M}-\mathrm{H}$, Mantel-Haenszel; CI, confidence interval; RR, risk ratio.

cated potential publication bias in the overall analysis $(\mathrm{p}=0.072$ and $\mathrm{p}=0.010$, respectively) (Fig. 3B).

\section{Seroconversion in NA-treated CHB patients}

Four of the selected studies examined viral seroconversion in
CHB patients treated with NAs. In our meta-analysis, we found no significant difference in the rates of seroconversion between patients treated with ETV and those who received LAM, LdT or TDF (RR, 0.98; 95\% CI, 0.80 to 1.20; p=0.84) (Fig. 3C). Heterogeneity in the seroconversion data was not detected among the 
B

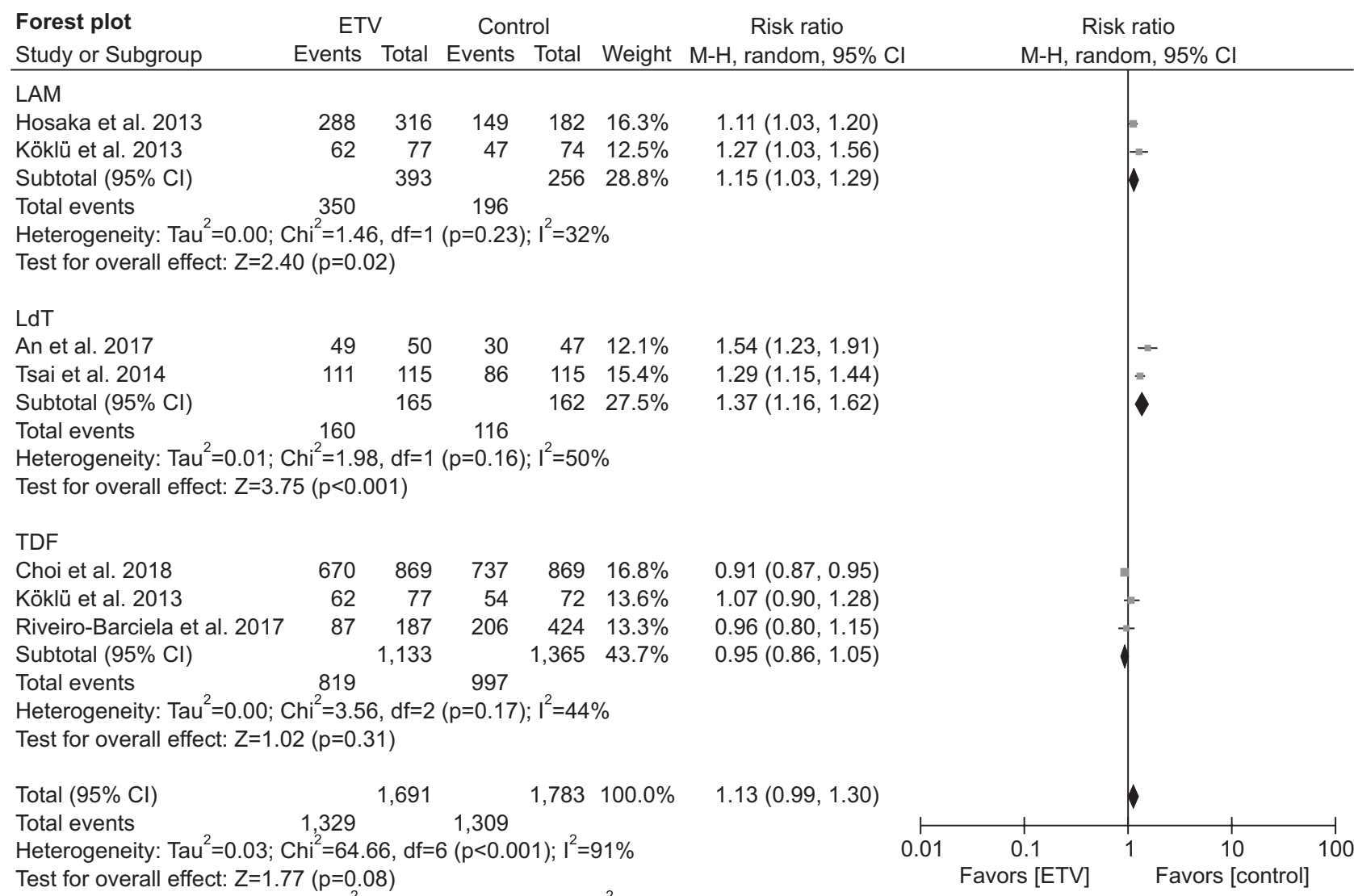

Test for subgroup differences: $\mathrm{Chi}^{2}=15.92, \mathrm{df}=2(\mathrm{p}=0.003) ; \mathrm{I}^{2}=87.4 \%$

\section{Funnel plot}

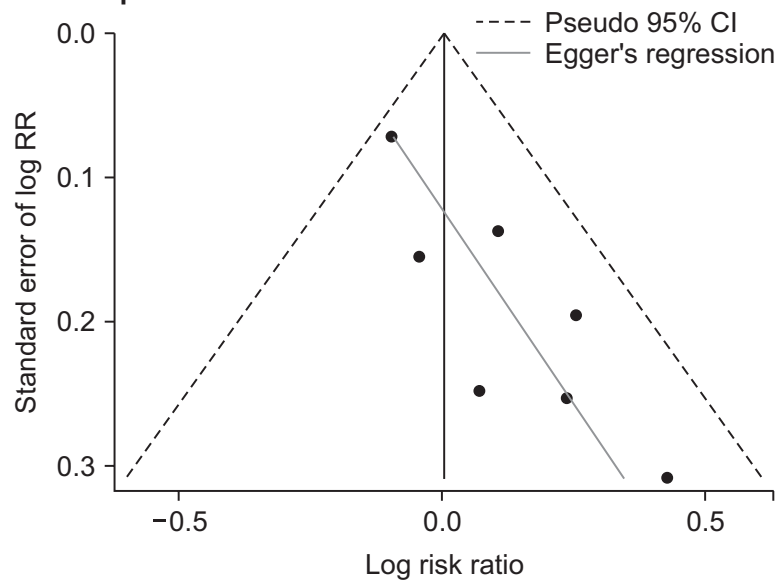

Fig. 3. Continued 1.

studies analyzed $\left(\mathrm{I}^{2}=0 \%, \mathrm{p}=0.54\right)$, but the result was not statistically significant. The Begg's and Egger's tests of the funnel plot data for the overall analysis did not indicate publication bias (p $>0.10$ for both) (Fig. 3C).

\section{Drug resistance in NA-treated CHB patients}

Six of the selected studies ${ }^{24,27,28,30,32,37}$ examined the incidence of NA resistance in CHB patients. Our meta-analysis showed that ETV treatment reduced the development of NA resistance by $95 \%$ (RR, 0.05; 95\% CI, 0.02 to $0.12 ; \mathrm{p}<0.001$ ), compared with that observed in patients receiving another NA (Fig. 3D). Subgroup analyses were performed because significant heterogeneity ( $\left.I^{2}=59 \%, p=0.02\right)$ was detected, which showed that the incidence of ETV resistance in CHB patients treated was 97\% and 96\% lower that of LAM (RR, 0.03; 95\% CI, 0.02 to 0.04 ; $\mathrm{p}<0.001$ ) or LdT (RR, 0.04; 95\% CI, 0.01 to 0.22 ; $\mathrm{p}<0.001$ ), re- 
C

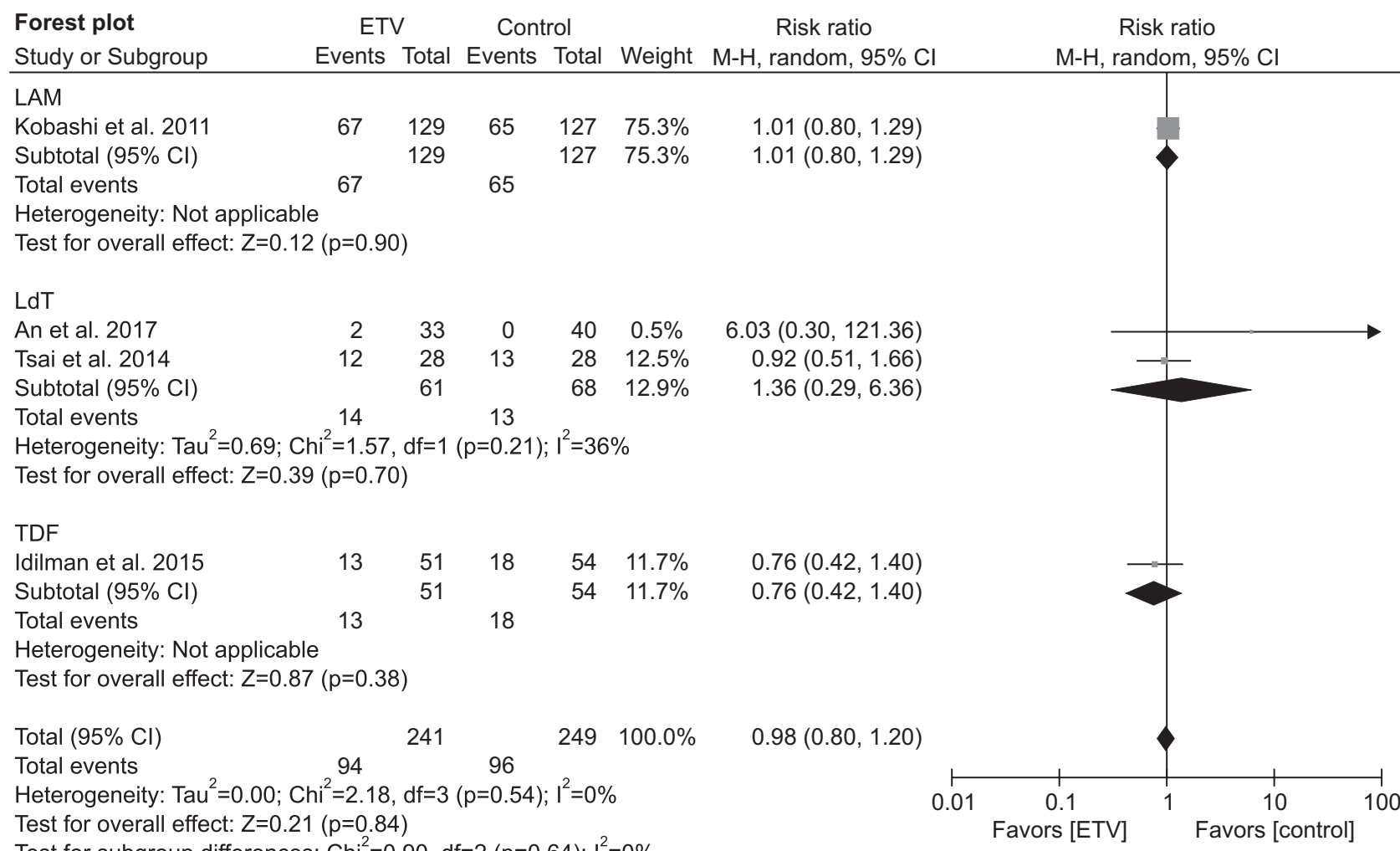

Test for subgroup differences: $\mathrm{Chi}^{2}=0.90, \mathrm{df}=2(\mathrm{p}=0.64) ; \mathrm{I}^{2}=0 \%$

\section{Funnel plot}

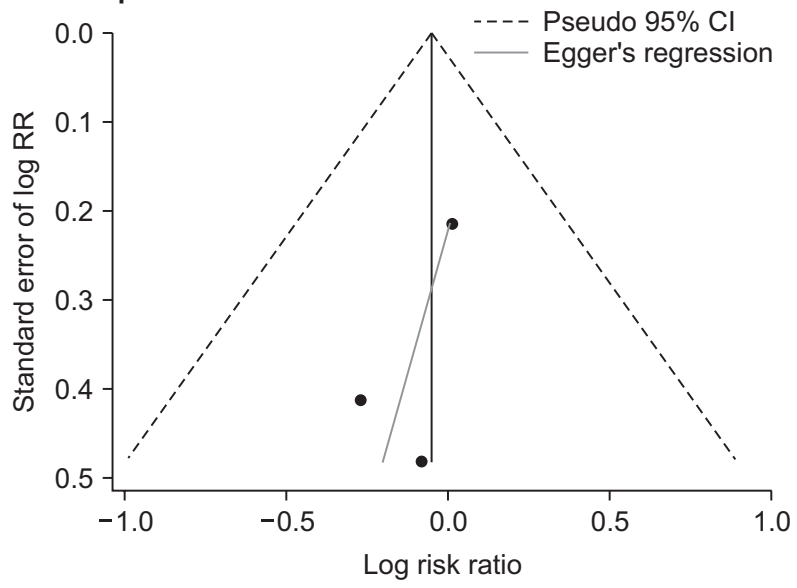

Fig. 3. Continued 2.

spectively. We found no significant difference in the rates of NA-resistance between CHB patients treated with ETV and those who were treated with TDF ( $p=0.95)$. The Begg's and Egger's tests of the funnel plot data for the overall analysis did not indicate publication bias ( $\mathrm{p}>0.10$ for both) (Fig. 3D).

\section{HCC incidence in NA-treated CHB patients with cirrhosis}

Seven of the selected studies ${ }^{24,27,29-32,35}$ reported the incidence of HCC in CHB patients with pre-existing cirrhosis who underwent NA treatment. Our meta-analysis found that the incidence of HCC in NA-treated patients with pre-existing cirrhosis was
5.49 times higher than that of those who did not have cirrhosis before initiating NA treatment (RR, 5.49; 95\% CI, 3.79 to 7.94; $\mathrm{p}<0.001$ ) (Fig. 4). Significant heterogeneity in the data regarding HCC in CHB patients with cirrhosis was not detected among the studies analyzed $\left(\mathrm{I}^{2}=22 \%, \mathrm{p}=0.26\right)$. The Begg's and Egger's tests of the funnel plot data did not indicate publication bias ( $p>0.10$ for both) (Fig. 4).

\section{DISCUSSION}

All NAs used in the treatment of CHB competitively bind 
D

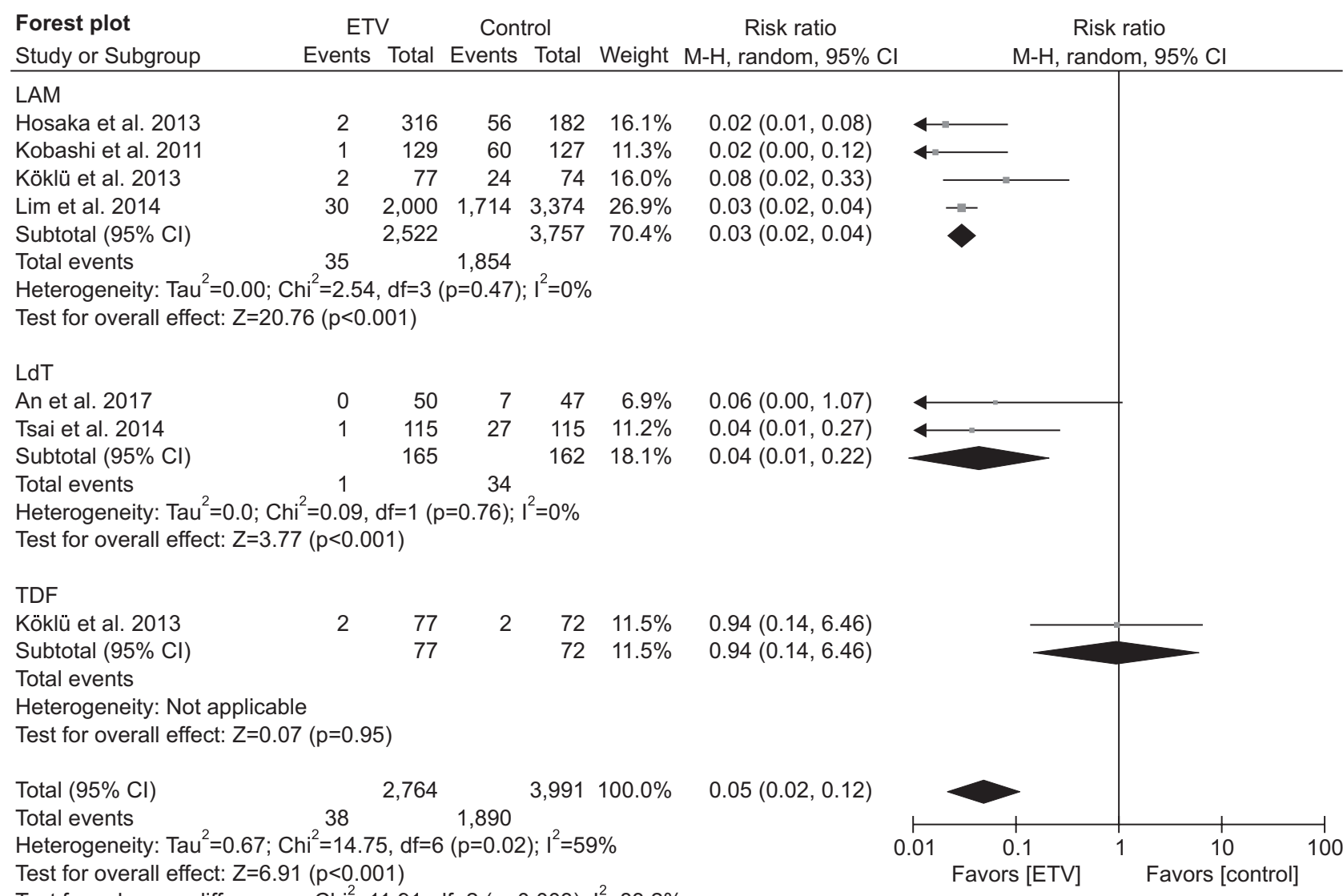

Test for subgroup differences: $\mathrm{Chi}^{2}=11.91, \mathrm{df}=2(p=0.003) ; \mathrm{I}^{2}=83.2 \%$

\section{Funnel plot}

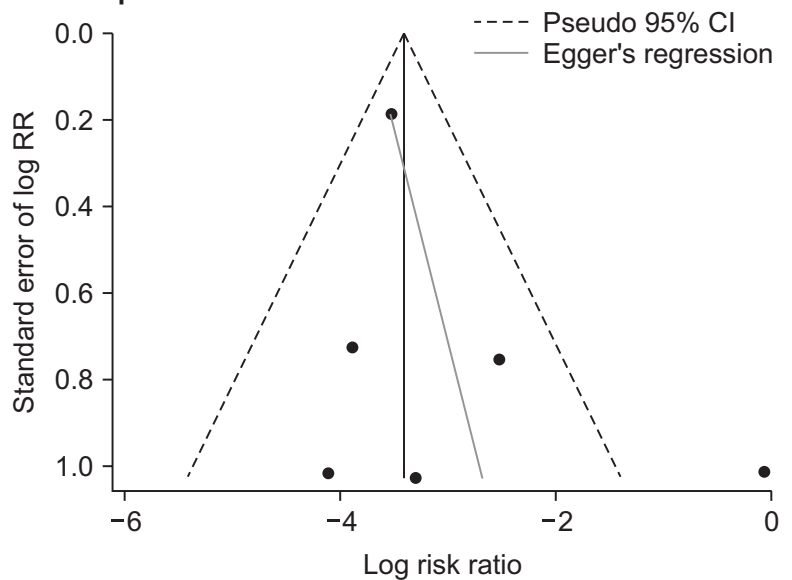

Fig. 3. Continued 3.

HBV DNA polymerase, which inhibits viral reverse transcription, thus blocking viral replication. However, NAs have no effect on the covalently closed circular DNA of HBV that remains stable for long periods in the nucleus of infected hepatocytes. ${ }^{43}$ If NA treatment is stopped before the viral DNA polymerase is inactivated or eliminated by cellular protein turnover, HBV levels rebound rapidly as the covalently closed circular DNA is used as template to produce viral transcripts which in turn serve as tem- plates for viral reverse transcription. ${ }^{44}$ Therefore, optimal CHB clinical outcome requires long-term suppression of HBV replication. Stemming the progression of cirrhosis and preventing HCC are additional primary clinical objectives that are intrinsically linked to viral suppression. However, whether different NAs have equivalent effects on HCC development is unclear, and the impact of cirrhosis on the protective effects of NAs has not been adequately characterized. 


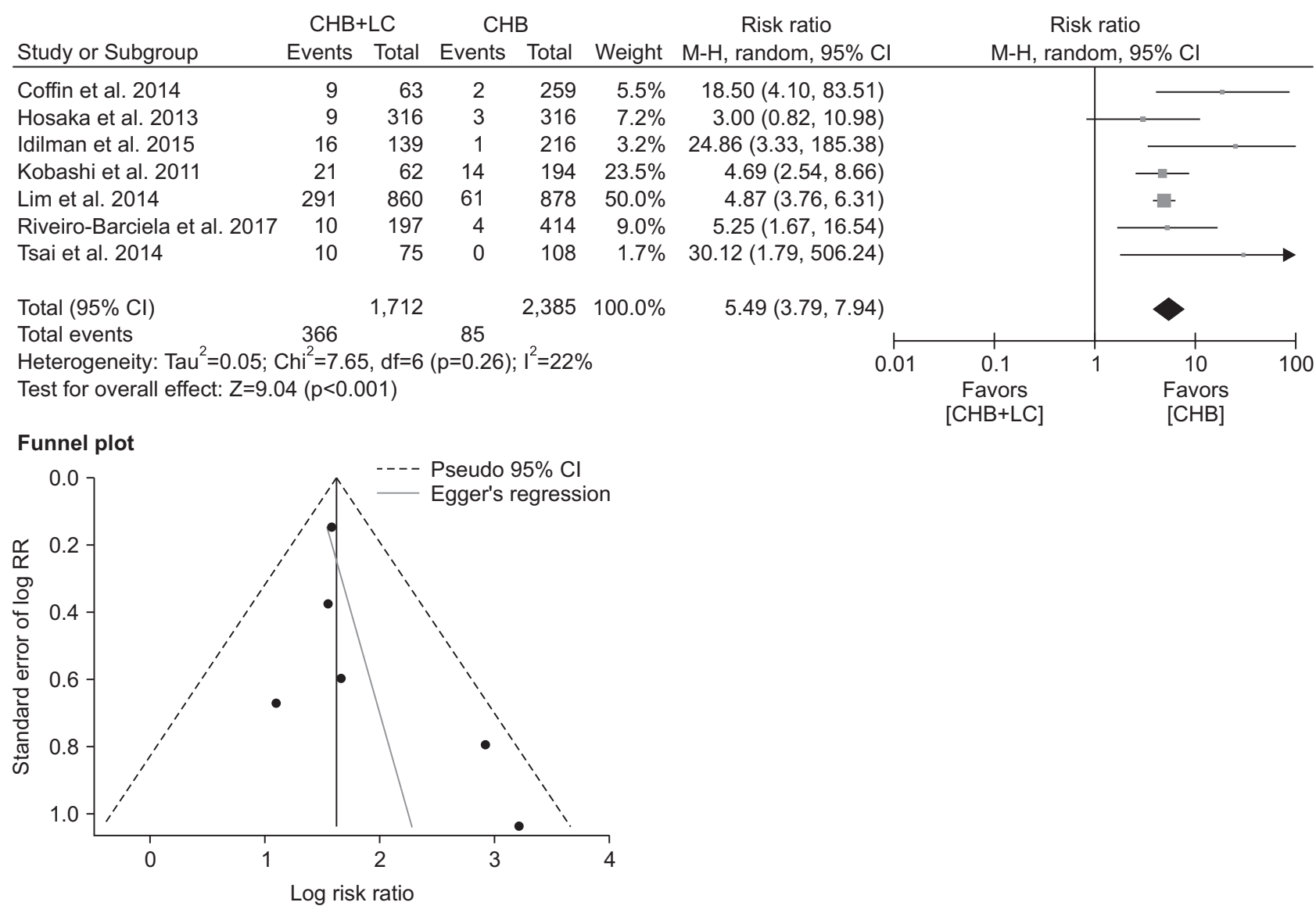

Fig. 4. Forest and funnel plots for the comparison of hepatocellular carcinoma incidence in NA-treated patients with chronic hepatitis B (CHB) and liver cirrhosis (LC) and CHB patients without liver cirrhosis (Begg's test: $\mathrm{z}=1.13, \mathrm{p}=0.260$; Egger's test: $\mathrm{t}=-1.24, \mathrm{p}=0.281$ ).

NA, nucleos(t)ide analogue; ETV, entecavir; M-H, Mantel-Haenszel; CI, confidence interval; LAM, lamivudine; LdT, telbivudine; TDF, tenofovir disoproxil fumarate; RR, risk ratio.

The current guidelines for CHB treatment recommend ETV, TDF, or TAF as first-line therapy. ${ }^{15,21,45}$ These recommendations are largely based on the incidence of drug resistance observed for the different NAs. Long-term use of LAM can result in drug resistance and viral breakthrough. ${ }^{46}$ Resistance against ADV and LdT monotherapies also occurs. ${ }^{21}$ The loss of HBV suppression due to resistance against these NAs requires rescue therapy in which either ETV or TDF is added to the treatment regimen. ${ }^{15,45,47}$ Although resistance to ETV is rare, certain viral mutations conferring LAM or LdT resistance can increase the risk of developing ETV resistance, ${ }^{21}$ and previous exposure to LAM has been shown to increase the risk of ETV resistance even in the absence of detectable LAM resistance. ${ }^{48}$ As a consequence, current guidelines recommend switching to TDF or adding ADV for rescue therapy in patients with LAM or LdT resistance, whereas ETV is recommended in patients with ADV resistance. ${ }^{45}$ In rare cases of ETV resistance, TDF should be added for antiviral rescue. Resistance to TDF has not been confirmed. With regard to selecting the best NA for reducing the risk of HCC, a low rate of drug resistance should be a prerequisite to ensure long-term suppression of viral replication. Our findings of our meta-anal- ysis regarding the incidences of drug resistance support these recommendations.

The results of our meta-analysis showed that the incidence of HCC in CHB patients treated with ETV was 21\% lower ( $p=0.34$ ) than that among patients treated with other NAs, which included TDF, LAM, ADV, and LdT. In the overall analysis, the only studies with RRs that did not favor ETV were those in which patients were treated with TDF (Fig. 2A). ${ }^{32,36,41,42}$ In the subgroup analysis, we analyzed the data from eight studies, ${ }^{29,31,32,35-37,41,42}$ and found no significant difference in HCC incidence between ETV and TDF ( $p=0.08$ ) (Fig. 2D). However, it is clear from the forest plot in Fig. 2A that the studies with the largest confidence intervals in the data set also had the smallest and greatest effect sizes (Coffin et al. ${ }^{35}$ and Hsu et al., ${ }^{36}$ respectively), which were likely confounding factors given that a sensitivity analysis could not isolate a single study as the source of heterogeneity (data not shown).

Our findings regarding HCC incidence for ETV versus TDF (Fig. 2D) are consistent with those of a study by Tsai et al. (2017). ${ }^{49}$ Their retrospective cohort study had a relatively large sample size $(n=546)$, but it was not selected our meta-analysis because 
they included patients also receiving medications that might influence the risk of HCC. Tsai et al ${ }^{49}$ reported that the cumulative incidence of HCC in ETV-treated patients $(n=359)$ was not significantly different than that in TDF-treated patients $(n=83)$. No RCTs of ETV versus TDF in a large sample have been reported, ${ }^{50}$ and no RCTs using TDF met our stringent study selection criteria. The results of our meta-analysis of studies comparing HCC incidence for ETV versus TDF highlight an important need for the implementation of standardized protocols for evaluating ETV and TDF efficacy in CHB patients, which has recently been voiced by others in the scientific community. ${ }^{50}$

Only three studies were included in our subgroup analysis of HCC incidence in ETV-treated versus that LdT-treated patients (Fig. 2C), and the combined sample of LdT-treated patients in our analysis was relatively small $(n=63)$. These may represent potential confounders of our results. Furthermore, we did not perform an analysis of publication bias due to the small number of studies in our ETV versus LdT subgroup analysis. However, our findings regarding HCC incidence for ETV versus LdT are also consistent with those of Tsai et al. ${ }^{49}$ They reported that the cumulative incidence of HCC in ETV-treated patients $(n=359)$ was not significantly different than that in LdT-treated patients $(n=104)$. We also found that the risk of HCC in ETV-treated patients was statistically similar to that in LdT-treated patients ( $\mathrm{p}>0.05)$.

Our subgroup analysis of HCC risk for ETV versus LAM treatments for CHB found that the risk of HCC in patients treated with ETV was 55\% lower than that of patients who were treated with LAM $(\mathrm{p}<0.001)$ (Fig. 2B). Studies in Asia and Europe have shown that LAM achieves HBV DNA reduction, HBeAg serological conversion, and alanine aminotransferase normalization, thereby delaying or preventing disease progression and reducing the incidence of HCC. ${ }^{51,52}$ Although the incidence of NA resistance is highest for LAM, the cost of LAM is approximately $80 \%$ to $86 \%$ lower than that of other NAs, and LAM may still be used as first-line treatment for CHB in some countries in an effort to maintain cost-effective government-provided health care. ${ }^{51,53}$ Although one study found that TDF monotherapy was more cost-effective for treating CHB than other NAs based on average annual and lifetime disease costs per patient, lifetime cost and life expectancy, and quality adjusted life years, ${ }^{54}$ a later study reported that cost-effectiveness of LAM plus ADV was similar to that of TDF. ${ }^{53}$ However, no combination of different NAs is currently recommended by the current guidelines, except for rescue therapy following the onset of drug resistance. ${ }^{14}$

Antiviral NA treatment is thought to indirectly reduce the risk of HCC by reducing HBV DNA load, improving liver inflammation, and promoting seroconversion of HBeAg. Therefore, we compared these clinical outcomes in ETV-treated CHB patients with those in CHB patients receiving other NAs. The overall analysis of hepatic biochemical response found that the incidence of favorable response was significantly greater among
ETV-treated patients ( $\mathrm{p}=0.04)$ (Fig. 3A), and the subgroup analysis showed that, while the rate of favorable biochemical response in ETV-treated patients was significantly higher than that of LAM-treated patients $(p<0.001)$, there was no significant difference between ETV and TDF or LdT. However, only one study was included in each of our analyses of LAM and LdT, which could have biased our results. In the overall and subgroup analyses, the effects of ETV on serological conversion were not significantly different from those of other NAs (Fig. 3C). However, LAM-treated patients were not included in these analyses, and only one study was used in the comparison of ETV and TDF, which might have confounded our analysis.

In our overall analysis, treatment with ETV increased the incidence of favorable virological response by 13\% (Fig. 3B), but this result was not statistically significant $(\mathrm{p}=0.08)$. While virological response in ETV-treated patients was significantly better than that in patients treated with LAM or LdT ( $\mathrm{p}<0.05$ for both), no significant difference in virological response was observed between ETV and TDF ( $p=0.31)$. Heterogeneity in the overall analysis of virological response was reduced in the subgroup analyses, but these results were not significant. Furthermore, the funnel plot analysis for virological response indicated a significant probability that publication bias affected our analysis. Therefore, with regard to virological response in CHB patients, our findings are not inconsistent with the recommendations of current $\mathrm{CHB}$ treatment guidelines suggesting either these two drugs for first-line monotherapy. ${ }^{14,15,21}$ However, like that observed in our analysis of HCC incidence, our lack of statistically significant findings of virological response for ETV versus TDF highlights the importance of developing and implementing standardized protocols in future studies in order to facilitate statistically conclusive analyses.

In a previous systematic review and Bayesian analysis of 20 RCTs of NA treatment for CHB, Woo et al. ${ }^{55}$ reported that TDFtreated patients had better rates of seroconversion and favorable virological and biochemical responses than those of patients treated with LAM, ETV, LdT, or ADV. They also reported that ETV was more effective for reducing HBV DNA level and normalizing alanine aminotransferase than were LAM, LdT, or $\mathrm{ADV}$, and that ETV was more effective for improving hepatic histological response than all other NAs. We did not include hepatic histological response in our meta-analysis of NA treatments because, in our experience, histological data quite often are not obtained during follow-up for NA treatment if favorable virological, immunological, and hepatic biochemical responses are achieved, except perhaps in RCTs. Furthermore, all of the RCTs included in the Woo et al. ${ }^{55}$ meta-analysis were published before 2010, and none of them performed a direct comparison of ETV and TDF. Our meta-analysis included studies published between 2011 and 2018, which should provide a better representation of clinicians increased experiences using NAs and improvements in the guidelines for $\mathrm{CHB}$ treatment. 
Multiple previous studies have found that pre-existing cirrhosis is an important risk factor for HCC in CHB patients receiving NA therapy. ${ }^{9,24,32,56}$ Previous studies show that NAs can halt the progression of liver cirrhosis histologically, prevent the occurrence of hepatic decompensation, and reduce the incidence of HCC by inhibiting virus replication. However, multiple studies have confirmed that HCC can develop in CHB patients despite effective HBV suppression. ${ }^{34,49,57,58}$ Our meta-analysis found that the incidence of HCC in NA-treated patients with pre-existing cirrhosis was 5.49 times higher than that of those without cirrhosis ( $<<0.001$ ) (Fig. 4). Therefore, future studies should place greater emphasis on subgroup analyses, especially with regard to elucidating the role of cirrhosis on long-term effects of ETV, TDF, and TAF treatments for CHB.

Although our findings show the reduction in HCC risk was statistically similar between ETV and TDF ( $p>0.05$ ), ETV may be preferred over TDF, due to the greater potential of TDF for adverse effects and perhaps a wider experience among physicians using ETV since its approval for CHB treatment in 2005, whereas TDF was approved in 2008. The tenofovir prodrug, TAF, was recently approved for treating CHB in the United States, Europe, and Japan, ${ }^{59}$ and has a lower incidence of renal toxicity and bone demineralization than TDF. ${ }^{60}$ However, very little longterm data is available for TAF with regard to HCC risk, ${ }^{10}$ so studies of TAF were not included in our analysis. Likewise, only 11 ADV-treated patients were included in our analysis, and we did not subject data for these patients to subgroup analysis.

Our findings are subject to certain additional limitations. Only one randomized controlled trial was included in our meta-analysis, whereas the remaining 12 studies were retrospective cohort studies. This might be viewed as a shortcoming, due to the greater potential for selection bias in retrospective studies, and study design has been shown to a crucial factor in the analysis of the effects of NAs on HCC risk. A previous systematic review and meta-analysis found that, while RCTs showed no significant benefit of NA treatment on HCC risk, case-control studies showed a significant benefit, and results for cohort studies showed an increased risk of HCC in NA-treated patients. Inconsistencies between the results of this previous meta-analysis and our findings present herein suggests that differences in study design might have contributed to the heterogeneity detected in our analysis of HCC incidence, as our meta-analysis included ten cohort studies and one RCT. Future large-scale RCTs with standardized methods are needed to better clarify the long-term effects of NAs on HCC risk, especially for comparisons of ETV versus TDF.

In the studies subjected to meta-analysis, we found no significant difference in HCC incidence, biochemical response, virological response, serological conversion, or drug resistance between ETV and TDF treatment groups ( $p>0.05$ ), which highlights the need for standardized protocols for future studies comparing ETV and TDF efficacy for reducing HCC risk and improving secondary outcomes. Our results did, however, show that ETV was superior to LAM with regard to HCC incidence, biochemical response, virological response, and drug resistance, and ETV was superior to LdT with regard to virological response and drug resistance ( $p<0.05$ for all). We also found that, despite the benefit of NA treatment, the incidence of HCC was higher in $\mathrm{CHB}$ patients with cirrhosis than in those without cirrhosis, which highlights the need for early detection and successful intervention using a high genetic barrier NA to avoid resistance and viral breakthrough. Our findings provide clinical guidance by forming a basis for the selection of optimal first-line antiviral therapies for CHB patients, and place an ever-growing burden on clinical researchers to design and implement higher quality studies of the effects of NA treatment on HCC risk.

\section{CONFLICTS OF INTEREST}

No potential conflict of interest relevant to this article was reported.

\section{ACKNOWLEDGEMENTS}

This study was supported by Application of Clinical Features of Capital City of Science and Technology Commission (grant number: Z171100001017082), Fund for Beijing Science \& Technology Development of TCM (grant number: JJ2016-14), Fund of Special research of TCM in Capital City (grant number: 17ZY02) and National Natural Science Foundation of China (grant number: 81874435).

\section{AUTHOR CONTRIBUTIONS}

Conception and design of the study: Y.J., Z.Y. Acquisition and analysis of data: Xinhui Wang, X.L., Z.D., L.Y. Statistical analysis: Xinhui Wang. Writing - original draft: Xinhui Wang, X.L. Writing - review and editing: Xianbo Wang, Z.Y. Approval of final version of the manuscript: all authors.

\section{ORCID}

Xinhui Wang

Xiaoli Liu

Zhibo Dang

Lihua $\mathrm{Yu}$

Yuyong Jiang

Xianbo Wang

Zhiyun Yang
https://orcid.org/0000-0001-7234-840X https://orcid.org/0000-0002-7267-0367 https://orcid.org/0000-0002-4550-8137 https://orcid.org/0000-0002-3228-8286 https://orcid.org/0000-0003-2011-3849 https://orcid.org/0000-0002-3593-5741 https://orcid.org/0000-0001-6389-3617

\section{REFERENCES}

1. Chen W, Zheng R, Baade PD, et al. Cancer statistics in China, 2015. CA Cancer J Clin 2016;66:115-132. 
2. Torre LA, Bray F, Siegel RL, Ferlay J, Lortet-Tieulent J, Jemal A. Global cancer statistics, 2012. CA Cancer J Clin 2015;65:87-108.

3. Ozakyol A. Global epidemiology of hepatocellular carcinoma (HCC epidemiology). J Gastrointest Cancer 2017;48:238-240.

4. Kew MC. Epidemiology of hepatocellular carcinoma in sub-Saharan Africa. Ann Hepatol 2013;12:173-182.

5. Venook AP, Papandreou C, Furuse J, de Guevara LL. The incidence and epidemiology of hepatocellular carcinoma: a global and regional perspective. Oncologist 2010;15 Suppl 4:5-13.

6. Centers for Disease Control and Prevention. CDC Health Information for International Travel 2014: The Yellow Book. New York: Oxford University Press, 2013.

7. Cui Y, Jia J. Update on epidemiology of hepatitis B and C in China. J Gastroenterol Hepatol 2013;28 Suppl 1:7-10.

8. Levrero M, Zucman-Rossi J. Mechanisms of HBV-induced hepatocellular carcinoma. J Hepatol 2016;64(1 Suppl):S84-S101.

9. Sohn W, Cho JY, Kim JH, et al. Risk score model for the development of hepatocellular carcinoma in treatment-naïve patients receiving oral antiviral treatment for chronic hepatitis B. Clin Mol Hepatol 2017;23:170-178.

10. Tang LSY, Covert E, Wilson E, Kottilil S. Chronic hepatitis B infection: a review. JAMA 2018;319:1802-1813.

11. Schoggins JW, Rice CM. Interferon-stimulated genes and their antiviral effector functions. Curr Opin Virol 2011;1:519-525.

12. Clark DN, Hu J. Hepatitis B virus reverse transcriptase: target of current antiviral therapy and future drug development. Antiviral Res 2015;123:132-137.

13. Sacks SL, Scullard GH, Pollard RB, Gregory PB, Robinson WS, Merigan TC. Antiviral treatment of chronic hepatitis B virus infection: pharmacokinetics and side effects of interferon and adenine arabinoside alone and in combination. Antimicrob Agents Chemother 1982;21:93-100.

14. Terrault NA, Bzowej NH, Chang KM, et al. AASLD guidelines for treatment of chronic hepatitis B. Hepatology 2016;63:261-283.

15. Liaw YF, Kao JH, Piratvisuth T, et al. Asian-Pacific consensus statement on the management of chronic hepatitis B: a 2012 update. Hepatol Int 2012;6:531-561.

16. Liaw YF, Sung JJ, Chow WC, et al. Lamivudine for patients with chronic hepatitis B and advanced liver disease. N Engl J Med 2004;351:1521-1531.

17. Lai CL, Yuen MF. Prevention of hepatitis B virus-related hepatocellular carcinoma with antiviral therapy. Hepatology 2013;57:399408.

18. Niro GA, Ippolito AM, Fontana R, et al. Long-term outcome of hepatitis B virus-related chronic hepatitis under protracted nucleos(t)ide analogues. J Viral Hepat 2013;20:502-509.

19. Wong GL, Tse YK, Yip TC, Chan HL, Tsoi KK, Wong VW. Longterm use of oral nucleos(t)ide analogues for chronic hepatitis B does not increase cancer risk: a cohort study of 44494 subjects. Aliment Pharmacol Ther 2017;45:1213-1224.

20. Lo AO, Wong GL. Current developments in nucleoside/nucleotide analogues for hepatitis B. Expert Rev Gastroenterol Hepatol
2014;8:607-622.

21. European Association for the Study of the Liver. EASL clinical practice guidelines: management of chronic hepatitis B virus infection. J Hepatol 2012;57:167-185.

22. Yin J, Li N, Han Y, et al. Effect of antiviral treatment with nucleotide/nucleoside analogs on postoperative prognosis of hepatitis $\mathrm{B}$ virus-related hepatocellular carcinoma: a two-stage longitudinal clinical study. J Clin Oncol 2013;31:3647-3655.

23. Wu CY, Lin JT, Ho HJ, et al. Association of nucleos(t)ide analogue therapy with reduced risk of hepatocellular carcinoma in patients with chronic hepatitis B: a nationwide cohort study. Gastroenterology 2014;147:143-151.

24. Hosaka T, Suzuki F, Kobayashi M, et al. Long-term entecavir treatment reduces hepatocellular carcinoma incidence in patients with hepatitis B virus infection. Hepatology 2013;58:98-107.

25. Sung JJ, Tsoi KK, Wong VW, Li KC, Chan HL. Meta-analysis: treatment of hepatitis B infection reduces risk of hepatocellular carcinoma. Aliment Pharmacol Ther 2008;28:1067-1077.

26. Wang JP, Kao FY, Wu CY, et al. Nucleos(t)ide analogues associated with a reduced risk of hepatocellular carcinoma in hepatitis B patients: a population-based cohort study. Cancer 2015;121:14461455.

27. Lim YS, Han S, Heo NY, Shim JH, Lee HC, Suh DJ. Mortality, liver transplantation, and hepatocellular carcinoma among patients with chronic hepatitis B treated with entecavir vs lamivudine. Gastroenterology 2014;147:152-161.

28. An J, Lim YS, Kim GA, et al. Telbivudine versus entecavir in patients with undetectable hepatitis B virus DNA: a randomized trial. BMC Gastroenterol 2017;17:15.

29. Idilman R, Gunsar F, Koruk M, et al. Long-term entecavir or tenofovir disoproxil fumarate therapy in treatment-naïve chronic hepatitis B patients in the real-world setting. J Viral Hepat 2015;22:504-510.

30. Kobashi H, Miyake Y, Ikeda F, et al. Long-term outcome and hepatocellular carcinoma development in chronic hepatitis B or cirrhosis patients after nucleoside analog treatment with entecavir or lamivudine. Hepatol Res 2011;41:405-416.

31. Riveiro-Barciela M, Tabernero D, Calleja JL, et al. Effectiveness and safety of entecavir or tenofovir in a Spanish cohort of chronic hepatitis B patients: validation of the Page-B score to predict hepatocellular carcinoma. Dig Dis Sci 2017;62:784-793.

32. Tsai MC, Chen $\mathrm{CH}$, Hung $\mathrm{CH}$, et al. A comparison of efficacy and safety of 2-year telbivudine and entecavir treatment in patients with chronic hepatitis B: a match-control study. Clin Microbiol Infect 2014;20:090-0100.

33. Papatheodoridis GV, Manolakopoulos S, Touloumi G, et al. Hepatocellular carcinoma risk in HBeAg-negative chronic hepatitis B patients with or without cirrhosis treated with entecavir: HepNet. Greece cohort. J Viral Hepat 2015;22:120-127.

34. Thiele M, Gluud LL, Dahl EK, Krag A. Antiviral therapy for prevention of hepatocellular carcinoma and mortality in chronic hepatitis B: systematic review and meta-analysis. BMJ Open 2013;3: 
e003265.

35. Coffin CS, Rezaeeaval M, Pang JX, et al. The incidence of hepatocellular carcinoma is reduced in patients with chronic hepatitis B on long-term nucleos(t)ide analogue therapy. Aliment Pharmacol Ther 2014;40:1262-1269.

36. Hsu YC, Wu CY, Lane HY, et al. Determinants of hepatocellular carcinoma in cirrhotic patients treated with nucleos(t)ide analogues for chronic hepatitis B. J Antimicrob Chemother 2014;69: 1920-1927.

37. Köklü S, Tuna Y, Gülșen MT, et al. Long-term efficacy and safety of lamivudine, entecavir, and tenofovir for treatment of hepatitis B virus-related cirrhosis. Clin Gastroenterol Hepatol 2013;11:8894.

38. Stang A. Critical evaluation of the Newcastle-Ottawa Scale for the assessment of the quality of nonrandomized studies in metaanalyses. Eur J Epidemiol 2010;25:603-605.

39. Jadad AR, Moore RA, Carroll D, et al. Assessing the quality of reports of randomized clinical trials: is blinding necessary? Control Clin Trials 1996;17:1-12.

40. Zeng X, Zhang Y, Kwong JS, et al. The methodological quality assessment tools for preclinical and clinical studies, systematic review and meta-analysis, and clinical practice guideline: a systematic review. J Evid Based Med 2015;8:2-10.

41. Choi J, Kim HJ, Lee J, Cho S, Ko MJ, Lim YS. Risk of hepatocellular carcinoma in patients treated with entecavir vs tenofovir for chronic hepatitis B: a Korean nationwide cohort study. JAMA Oncol 2019;5:30-36.

42. Kim BG, Park NH, Lee SB, et al. Mortality, liver transplantation and hepatic complications in patients with treatment-naïve chronic hepatitis B treated with entecavir vs tenofovir. J Viral Hepat 2018;25:1565-1575.

43. Nassal M. HBV cccDNA: viral persistence reservoir and key obstacle for a cure of chronic hepatitis B. Gut 2015;64:1972-1984.

44. Wong DK, Seto WK, Fung J, et al. Reduction of hepatitis B surface antigen and covalently closed circular DNA by nucleos(t)ide analogues of different potency. Clin Gastroenterol Hepatol 2013;11: 1004-1010.

45. Terrault NA, Lok ASF, McMahon BJ, et al. Update on prevention, diagnosis, and treatment of chronic hepatitis B: AASLD 2018 hepatitis B guidance. Hepatology 2018;67:1560-1599.

46. Lingala S, Lau DT, Koh C, Auh S, Ghany MG, Hoofnagle JH. Long-term lamivudine therapy in chronic hepatitis B. Aliment Pharmacol Ther 2016;44:380-389.

47. Li W, Wang L, Liu Y, Li Y, Yu S. Efficacy of entecavir-based rescue therapy in lamivudine-resistant chronic hepatitis B patients in China: a retrospective study. Pharmazie 2018;73:223-247.
48. Lee JH, Cho Y, Lee DH, et al. Prior exposure to lamivudine increases entecavir resistance risk in chronic hepatitis B Patients without detectable lamivudine resistance. Antimicrob Agents Chemother 2014;58:1730-1737.

49. Tsai MC, Chen CH, Hu TH, et al. Long-term outcomes of hepatitis $\mathrm{B}$ virus-related cirrhosis treated with nucleos(t)ide analogs. J Formos Med Assoc 2017;116:512-521.

50. Flemming JA, Terrault NA. Tenofovir vs entecavir for hepatocellular carcinoma prevention in patients with chronic hepatitis $\mathrm{B}$ : one of these things is not like the other. JAMA Oncol 2019;5:17-18.

51. Yao GB, Zhu M, Cui ZY, Wang BE, Yao JL, Zeng MD. A 7-year study of lamivudine therapy for hepatitis B virus e antigen-positive chronic hepatitis B patients in China. J Dig Dis 2009;10:131137.

52. Xu B, Lin L, Xu G, et al. Long-term lamivudine treatment achieves regression of advanced liver fibrosis/cirrhosis in patients with chronic hepatitis B. J Gastroenterol Hepatol 2015;30:372-378.

53. Jayakumar R, Joshi YK, Singh S. Laboratory evaluation of three regimens of treatment of chronic hepatitis $\mathrm{B}$ : tenofovir, entecavir and combination of lamivudine and adefovir. J Lab Physicians 2012;4:10-16.

54. Buti M, Brosa M, Casado MA, Rueda M, Esteban R. Modeling the cost-effectiveness of different oral antiviral therapies in patients with chronic hepatitis B. J Hepatol 2009;51:640-646.

55. Woo G, Tomlinson G, Nishikawa Y, et al. Tenofovir and entecavir are the most effective antiviral agents for chronic hepatitis B: a systematic review and Bayesian meta-analyses. Gastroenterology 2010;139:1218-1229.

56. Papatheodoridis GV, Chan HL, Hansen BE, Janssen HL, Lampertico P. Risk of hepatocellular carcinoma in chronic hepatitis B: assessment and modification with current antiviral therapy. J Hepatol 2015;62:956-967.

57. Wong GL, Tse YK, Chan HL, Yip TC, Tsoi KK, Wong VW. Oral nucleos(t)ide analogues reduce recurrence and death in chronic hepatitis B-related hepatocellular carcinoma. Aliment Pharmacol Ther 2016;43:802-813.

58. Honda M, Shirasaki T, Terashima T, et al. Hepatitis B virus (HBV) core-related antigen during nucleos(t)ide analog therapy is related to intra-hepatic HBV replication and development of hepatocellular carcinoma. J Infect Dis 2016;213:1096-1106.

59. Ogawa E, Furusyo N, Nguyen MH. Tenofovir alafenamide in the treatment of chronic hepatitis B: design, development, and place in therapy. Drug Des Devel Ther 2017;11:3197-3204.

60. Scott LJ, Chan HLY. Tenofovir alafenamide: a review in chronic hepatitis B. Drugs 2017;77:1017-1028. 